\title{
Electrophysiological assessment of optic nerve disease
}

\begin{abstract}
The electrophysiological findings in optic nerve and primary ganglion cell dysfunction are reviewed. The value of the pattern reversal visual-evoked potential (VEP) in the diagnosis of optic nerve disease, and the pattern appearance VEP in the demonstration of the intracranial misrouting associated with albinism, are discussed. The pattern electroretinogram (PERG) is used in the direct assessment of ganglion cell function. The use of PERG or multifocal electroretinography (mfERG), to enable the distinction between VEP delay due to optic nerve disease and that due to macular dysfunction, is described. Eye (2004) 18, 1133-1143. doi:10.1038/sj.eye.6701573

Keywords: electroretinogram; pattern ERG; visual-evoked potential; macula; albinism; optic atrophy
\end{abstract}

\section{Fundamentals}

\section{Visual-evoked cortical potential (VEP)}

The visual-evoked cortical potential (VEP) is an important electrophysiological test in the investigation of suspected optic nerve disease. The stimulus for diagnostic VEP is usually a reversing black and white checkerboard or grating (PVEP), but an appearance stimulus (onset/offset) can also be used. The latter stimulus is of particular value in demonstrating the misrouting of optic nerve and chiasmal fibres that occurs in ocular or oculocutaneous albinism. Diffuse flash stimulation has a role, but the flash VEP (FVEP) is less sensitive to the effects of disease than the pattern VEP, and is highly variable across a population. However, due to its low interocular or interhemispheric asymmetry in a normal subject, the FVEP may detect interocular or interhemispheric asymmetry within an individual patient.

The pattern reversal VEP (see Figure 1) consists of a prominent positive component at
GE Holder

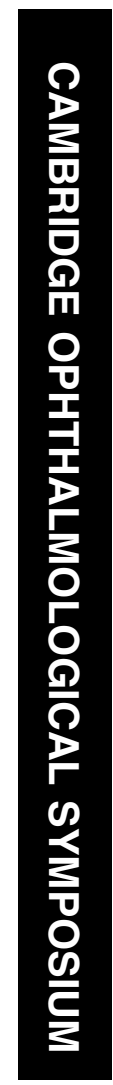

approximately $100 \mathrm{~ms}$ (P100) preceded and followed by negative components (N75 and N135). Analysis concentrates on the implicit time (usually termed latency) and amplitude of P100. In addition to the detection of optic nerve dysfunction, chiasmal and retrochiasmal dysfunction can be assessed by examining the distribution of the VEP over the posterior scalp. Although a delayed P100 component often occurs in association with optic nerve disease, delays are also commonplace in macular dysfunction, and a delayed VEP should not be considered pathognomonic of optic nerve disease. An associated test of macular function, such as the pattern electroretinogram (PERG) or multifocal ERG (mfERG) allows an improved interpretation of an abnormal VEP (see Holder ${ }^{1}$ for a recent review).

\section{Pattern electroretinogram}

Pattern electroretinography (PERG) is an established technique for the objective assessment of central retinal function. ${ }^{2,3} \mathrm{~A}$ reversing checkerboard, similar to that used for the PVEP, evokes the small electrical potentials that largely arise from inner retina. The normal PERG, using techniques recommended by the International Society for Clinical Electrophysiology of Vision (ISCEV), ${ }^{4}$ is recorded using corneal electrodes that do not interfere with the optics of the eye. It consists of a prominent positive component at approximately $50 \mathrm{~ms}$ and a larger negativity at approximately $95 \mathrm{~ms}$ (eg Figure 1). These components are known as P50 and N95 according to conventional neurophysiological practice whereby a component is identified by its polarity and approximate latency. ${ }^{5}$ The exact origins of these components have not been identified at the time of writing, but it seems that N95 is generated in relation to retinal ganglion cell function. Some of P50 appears to be generated more distally, but perhaps $70 \%$ of P50 also has origins in relation to spiking cell function. ${ }^{2,6}$ Even though the PERG has inner
Moorfields Eye Hospital London, UK

Correspondence: GE Holder Moorfields Eye Hospital City Road London EC1V 2PD UK

Tel: + 442075662120

Fax: +442075662556 E-mail: graham.holder@ moorfields.nhs.uk

Received: 4 September 2003

Accepted: 4 September 2003 

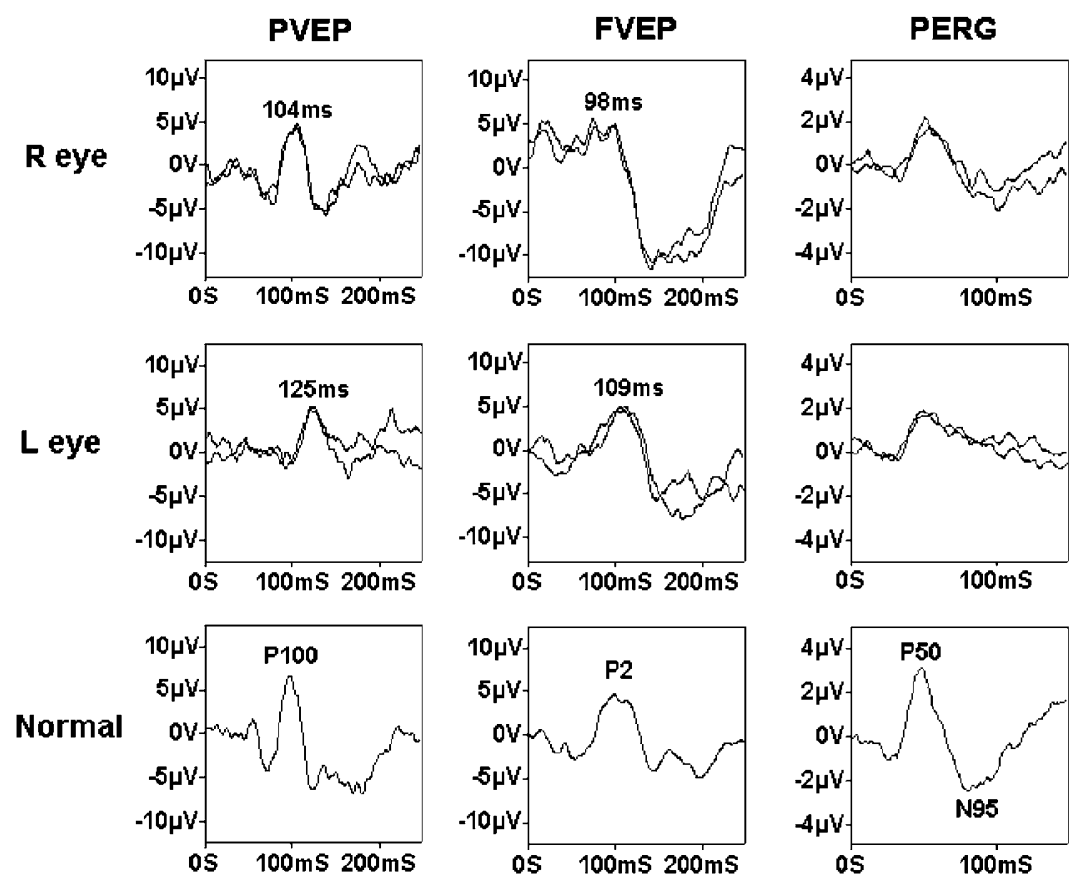

Figure 1 Delayed VEPs 7 months following left optic neuritis in a 39-year-old male. Note that the delay is present in both pattern and flash VEPs, and that there is selective reduction in the N95 component from the affected eye, in keeping with retrograde degeneration to the retinal ganglion cells. Right eye findings show no significant abnormality. Visual acuities were $6 / 6$ right; 6/12 left.

retinal origins, the P50 component is 'driven' by the macular photoreceptors and can thus be used as an index of macular function. A 'steady state' waveform is obtained if a rapid $(>3.5 \mathrm{~Hz})$ stimulus rate is used; however, this does not allow measurement of individual components and ISCEV recommends the transient PERG for routine diagnostic work.

\section{Electroretinogram}

Although not of direct importance in the assessment of optic nerve disease, given the possible difficulties in distinguishing clinically between disturbance of optic nerve and macular function, and the possibility that such macular dysfunction may be secondary to generalised retinal dysfunction, a brief description of (full-field) electroretinogram (ERG) is warranted. The ERG is the mass response of the retina, usually to a diffuse shortduration flash delivered via a Ganzfeld bowl. It is recorded, as is the PERG, using corneal electrodes. The main components of the ERG are the negative going awave and the positive going b-wave. The a-wave, in response to a bright flash in a dark-adapted eye, largely reflects photoreceptor function, but there may be a contribution from postreceptoral structures, ${ }^{7,8}$ particularly with low stimulus luminance. The b-wave, which is of higher amplitude than the a-wave in normals, reflects postphototransduction activity. It is largely produced in relation to ON- (depolarising) bipolar cell function. $^{8-10}$ The ISCEV Standard ERG ${ }^{11}$ incorporates a rod-specific response to a dim light under scotopic conditions, and a 'Standard; mixed rod-cone response to a bright white flash under dark adaptation. This latter response is dominated by rod function. A recent recommendation is an additional response to a brighter flash. The maximal ERGs shown below utilise this stimulus better to demonstrate the a-wave. Photopic ERGs are recorded both to a single flash (with adequate photopic adaptation and a rod-suppressing background) and to a $30 \mathrm{~Hz}$ flicker stimulus; rods are unable to respond to a $30 \mathrm{~Hz}$ stimulus due to poor temporal resolution. The ERG is a mass response and is therefore normal when dysfunction is confined to small retinal areas. This also applies to macular dysfunction; despite the high photoreceptor density, an eye with purely macular disease has a normal ERG.

\section{Multifocal electroretinogram}

The multifocal electroretinogram (mfERG) is a relatively new technique ${ }^{12}$ providing simultaneous assessment of local retinal areas using a pseudorandom binary sequence stimulation technique. The stimulus usually consists of black and white hexagons covering approximately $50^{\circ}$. The mfERG can therefore provide an index of central retinal function that extends the data 

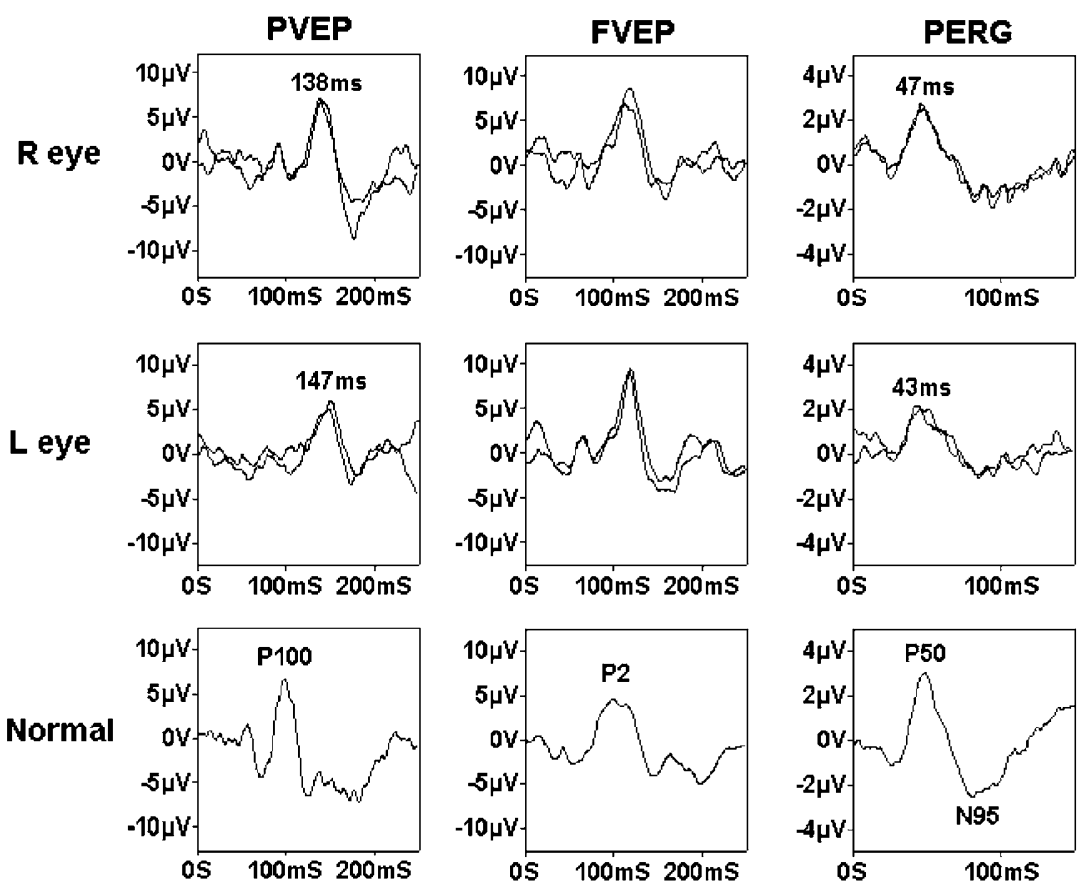

Figure 2 This 37-year-old female complained of blurred vision in her left eye following exercise or a hot bath. Other than questionable pallor of the left optic disc, examination was unremarkable. Visual acuities were $6 / 5$ bilaterally. The symptomatic left eye shows VEP evidence of marked optic nerve conduction delay; here is also reduction in the left eye PERG N95 component with shortening of P50 component latency. Note the marked subclinical delay in right optic nerve conduction, presumably explaining the lack of a relative afferent pupillary defect in relation to the left eye.

provided by the PERG by giving additional spatial information, and possibly layer localisation within the retina. ${ }^{3}$ However, in the context of suspected optic nerve disease the PERG has two main advantages. Firstly, the N95 component of the PERG provides a direct assessment of ganglion cell function. Secondly, in relation to VEP interpretation, the PERG is evoked by a similar stimulus (a reversing checkerboard), and is thus more satisfactory than the luminance-related mfERG. The reader is referred elsewhere ${ }^{13}$ for more details of clinical applications of mfERG.

\section{Clinical applications}

\section{Optic nerve demyelination}

After early clinical studies using VEPs evoked by diffuse flash stimuli (FVEP), ${ }^{14}$ a major breakthrough occurred with a series of publications from the Queen Square group in London, led by Martin Halliday. Not only was it demonstrated that the PVEP is delayed in patients with optic neuritis, but also that the PVEP delay persists following clinical recovery. ${ }^{15}$ They further demonstrated that the VEP could reveal optic nerve conduction delay in the absence of any signs or symptoms of clinical optic nerve involvement. ${ }^{16}$ Usually, VEPs showed conduction delay with less marked amplitude change. There was a stronger relationship between visual acuity and any amplitude change than with the magnitude of the conduction delay. Many authors (see Holder ${ }^{17}$ for a review) subsequently confirmed the nature of these VEP findings. Typical examples appear in Figures 1 and 2. The VEPs in some patients continue to improve for some considerable time after the acute episode. This has been ascribed to remyelination of demyelinated nerve fibres. $^{18}$

In the $1970 \mathrm{~s}$, prior to the development of computerised tomographic scanning (CT) or magnetic resonance imaging (MRI), invasive neuroradiology such as myelography was the investigation of choice for patients presenting with spinal cord lesions. The ability of the VEP to detect subclinical optic nerve demyelination thus had a profound impact on the management of such patients, the demonstration of optic nerve conduction delay obviating the need for myelography by identifying dissemination of lesions. Initially, when MRI became readily available, referrals for VEP in patients with suspected multiple sclerosis reduced. However, as it is now apparent that the specificity of the changes on MRI may be less than originally anticipated, the VEP continues to be of value in the diagnosis of demyelination. 
The PERG is abnormal in approximately $40 \%$ of patients with optic nerve demyelination, but in $85 \%$ of those patients the abnormality is confined to the N95 component, in keeping with retrograde degeneration to the retinal ganglion cells. ${ }^{19}$ A small percentage of patients show P50 component involvement, but then the reduction in P50 component amplitude may be accompanied by a shortening of P50 latency (eg Figure 2). P50 latency increase is not a feature of optic nerve or retinal ganglion cell disease.

It should be noted that the findings in acute optic neuritis, for example within 7 days of the onset of symptoms, differ from those in long-standing disease. PVEP amplitude shows profound reduction, but there is much less pronounced latency change. In addition, there is a reduction in the P50 component of the PERG. This acute effect on P50 tends to resolve, and as P50 amplitude returns (usually within a few weeks) the amplitude of the PVEP also improves. However, a greater PVEP latency delay is observed synchronous with the improvement in PERG P50 and PVEP amplitude. ${ }^{19,20}$ At presentation, N95 reduction is concomitant to that in $\mathrm{P} 50$, with a similar N95: P50 ratio to that in the unaffected eye; a PERG N95 component abnormality may be present after the acute phase has resolved. Youl et $a l^{21}$ described marked reduction in PVEP amplitude during the acute phase accompanied by gadolinium enhancement of the optic nerve lesion on MRI, in keeping with inflammation. There was significant increase in PVEP amplitude with resolution of lesion enhancement. It is postulated that the recovery of VEP amplitude is associated with resolution of oedema, and the increasing delay with demyelination. A later MRI/VEP study reported that poor visual acuity and decreased VEP amplitude were associated with atrophy demonstrated by imaging. ${ }^{22}$ In a recent report of acute optic neuritis, eight of 17 eyes had no detectable PVEP at presentation, but this was not a predictor of final visual acuity. ${ }^{1}$ However, it was noted the only two eyes not to regain 6/12 acuity or better at follow-up had PERG P50 amplitudes of $<0.5 \mu \mathrm{V}$ at presentation (normal $>2.0 \mu \mathrm{V})$. A larger series is necessary before firm prognostic conclusions can be drawn.

Newer techniques have been used further to investigate the nature of the VEP abnormalities in optic nerve demyelination. Hood et $a l^{23}$ used multifocal VEP (mfVEP) techniques to demonstrate local areas of optic nerve damage following optic neuritis. In the acute phase, all the three patients examined had marked visual field defects, and reduced mfVEPs in regions of poor field sensitivity. By 4-7 weeks, fields and mfVEP amplitudes had recovered, but substantial mfVEP delays were present at many locations. Jones and colleagues ${ }^{24}$ used conventional reversal VEPs, but with an elegant stimulus design such that separate responses were recorded during the same session to stimulation of central, nasal, and temporal regions of the macula. They concluded that central fibres were most affected by demyelination.

\section{Ischaemic optic neuropathy}

Wilson ${ }^{25}$ provided the first detailed analysis of the electrophysiological findings in nonarteritic ischaemic optic neuropathy (NAION), although 'delays' in the PVEP had previously been reported. ${ }^{26}$ Both PVEP and flash VEPs (FVEPs) were examined in a mixed group of 15 arteritic and nonarteritic patients. The predominant abnormality was amplitude reduction; only four patients showed latency changes ( $<10 \mathrm{~ms}$ delay). The VEPs were invariably normal in the clinically uninvolved eye. The findings were contrasted with those in optic nerve demyelination where there is often subclinical involvement of the fellow eye and where latency delays in excess of $10 \mathrm{~ms}$ are common. The high incidence of normal latency VEPs of reduced amplitude was confirmed by other authors, ${ }^{27-31}$ although Glaser and Laflamme found PVEP delays in acute cases. Harding's group noted that eyes with a delayed or triphasic FVEP had temporal arteritis. The FVEP delay in arteritic ischaemic optic neuropathy was confirmed by this author, ${ }^{29}$ who further found the PVEP more sensitive than the FVEP in NAION. Amplitude reductions were usually relative to the uninvolved eye. Cox et $a l^{30}$ contrasted the PVEPs from 24 NAION eyes with 22 optic nerve demyelination eyes. There was a mean latency difference of $21 \mathrm{~ms}$ between the involved and the uninvolved eyes in demyelination, but only $3 \mathrm{~ms}$ for NAION. Wildberger ${ }^{31}$ found that patients with an inferior altitudinal defect touching the horizontal meridian showed apparent latency delays attributable to preservation of the normal longer latency response from the superior field. ${ }^{32}$ Thompson et $a l^{33}$ found 'delays' in some cases that could be explained by complete or partial substitution of the paramacular P135 subcomponent for the usually dominant, macularderived P100 component. They emphasised the difficulties in accurate component identification with a single midline recording channel and a large stimulus field (eg $15^{\circ}$ radius).

The PERG has received relatively little attention in ischaemic optic neuropathy. N95 reduction may occur in ischaemic optic neuropathy (ION), 5,34,35 but the P50 component of the PERG is more frequently affected in ION than demyelination, ${ }^{34}$ perhaps reflecting more widespread vascular-related dysfunction anterior to the retinal ganglion cells. A report showing histopathological change in both inner and outer nuclear layers of the retina in ION has appeared. ${ }^{36}$ 


\section{Optic nerve compression}

Although tumours such as sphenoid wing meningiomata can cause optic nerve compression, probably the most common cause is asymmetrical extension of a pituitary tumour from the pituitary fossa. Following the initial report by Muller ${ }^{37}$ that the flash VEP could be of abnormal latency in chiasmal dysfunction, others noted that the maximum abnormality localised contralateral to the visual field defect, ${ }^{38-40}$ as would be predicted. The first reports using contrast stimuli appeared in 1976. Van Lith's group $^{41}$ used both full and hemifield steady-state $(8 \mathrm{~Hz})$ stimulation in six patients with bitemporal hemianopia, reporting both phase and amplitude abnormalities contralateral to the stimulated eye.

Halliday et $a l^{42}$ provided the first detailed report of transient PVEP. They found markedly asymmetrical scalp distribution in 10 patients with chiasmal dysfunction using a $16^{\circ}$ radius, 50 -min check stimulus. In particular, they first described a 'crossed' VEP asymmetry, typically found in chiasmal dysfunction, where the findings from one eye are more abnormal over one hemisphere, but asymmetrical distribution reverses when the fellow eye is stimulated. Unexpectedly, they reported 'paradoxical' lateralisation, such that maximum abnormality was localised ipsilateral to the visual field defect. PVEP abnormalities were present in some eyes with normal visual fields (kinetic). The findings often showed distortion of the waveform, and although there were latency delays, these were of less magnitude than regularly seen in demyelination where, additionally, symmetry across the scalp was much more frequent. The use of hemifield stimulation was discussed in another publication by the same group. ${ }^{43}$

This 'crossed' asymmetry was confirmed by Holder ${ }^{44}$ in 10 patients, but when using full-field stimulation with a small field, small check stimulus ( $\left.11^{\circ} / 26 \mathrm{~min}\right)$ the PVEP abnormality was contralateral to the field defect rather than 'paradoxical'. Although apparently contradictory, the alternate abnormality lateralisation merely reflects the use of a smaller stimulating field and check size, paradoxical lateralisation only being a feature of recordings using a large field stimulus. It was confirmed that the asymmetrical scalp distribution was not typical for demyelination. Although abnormal VEPs could occur in eyes with full visual fields, normal PVEPs could occur in eyes with field defects. Latency delays were common. Those findings were extended in a report of 34 patients with histologically confirmed nonfunctioning chromophobe adenomas. ${ }^{45}$ The PVEPs could indicate marked functional asymmetry when CT scan suggested symmetrical midline suprasellar extension. The PVEPs were usually more sensitive than visual acuity and visual fields. Similar findings subsequently confirmed the 'crossed' PVEP asymmetry to be pathognomonic of chiasmal dysfunction. ${ }^{46-52}$

PERG abnormalities may also occur due to retrograde degeneration to the retinal ganglion cells. ${ }^{2}$ Some reports have appeared suggesting the PERG to be a useful prognostic indicator for visual outcome in the preoperative assessment of pituitary tumour. ${ }^{53,54}$ That suggestion was confirmed by Parmar et $a l{ }_{;}^{55}$ an abnormal preoperative PERG correlated with a lack of postoperative recovery.

\section{Primary disorders of retinal ganglion cell function}

Leber hereditary optic neuropathy (LHON) and Kjertype dominant optic atrophy (DOA) ${ }^{56}$ are the two most commonly occurring examples of primary ganglion cell disease. LHON usually presents with painless sequential bilateral visual loss and is related to mutation in the mitochondrial genome. Females are less frequently affected than males, but the reason for this is not clear. As there is incomplete penetrance, there are probably other determining factors. Most patients are between 11 and 30 years of age at presentation, but earlier and much later age of onset can occur. ${ }^{57,58}$ Currently, genetically confirmed diagnoses have been established in patients as young as 2 and as old as 80 years of age ${ }^{59}$ (NJ Newman, personal communication). There may be disc swelling at presentation, often accompanied by microangiopathic changes in the disc vessels, but there is no fluorescein leakage from the disc even in the late phase of fundus fluorescein angiography. PVEPs, when detectable, are usually markedly abnormal with both delay and waveform distortion, but there is marked reduction in the N95 component of the PERG. ${ }^{60}$ One report has suggested, in the 11778 mutation, that VEP abnormalities may precede the onset of symptoms. ${ }^{61}$

Kjer-type DOA is related to mutation in OPA1 on chromosome 3 . There is usually progressive visual acuity loss associated with disc pallor, a centrocaecal visual field defect and defective colour vision. ${ }^{62}$

Histopathological ${ }^{63,64}$ and electrophysiological studies $^{5,65,66}$ are in keeping with degeneration of the retinal ganglion cells leading to optic atrophy. As with LHON, VEPs are often delayed, but there tends to be better preservation of the waveform in early disease. Again, in keeping with ganglion cell dysfunction, there is N95 reduction in the PERG, and indeed the PERG can occasionally be profoundly abnormal even in the absence of marked PVEP abnormality (eg Figure 3). PERG abnormalities confined to N95 were reported in younger patients in a recent study of 13 patients from 8 families. ${ }^{66}$ In more advanced disease, additional P50 component involvement occurred; there was P50 amplitude reduction and shortening of P50 latency in severe 


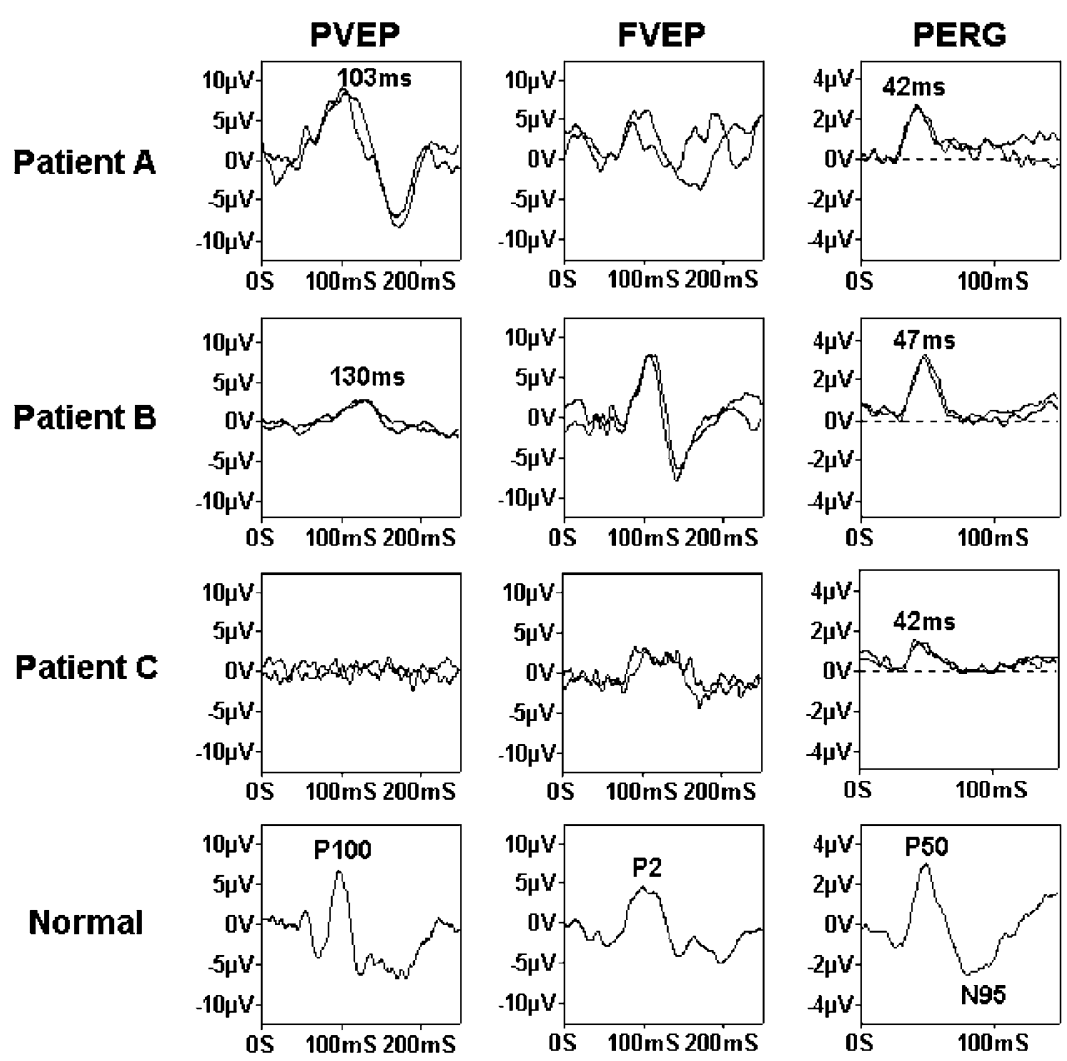

Figure 3 VEP and PERG findings from three patients with dominantly inherited optic atrophy. Patient A (VA 6/18) has a markedly abnormal PERG, with clear N95 component reduction and shortening of P50 component latency, but a VEP that falls within the normal range. Patient B (VA 6/9) shows clear VEP delay, despite the better visual acuity, and has PERG abnormality confined to N95. Patient C (VA HM), with end-stage disease, had no detectable PVEP. PERG shows loss of N95 with shortening of P50 latency and additional P50 amplitude abnormality $(<2 \mu \mathrm{V})$.

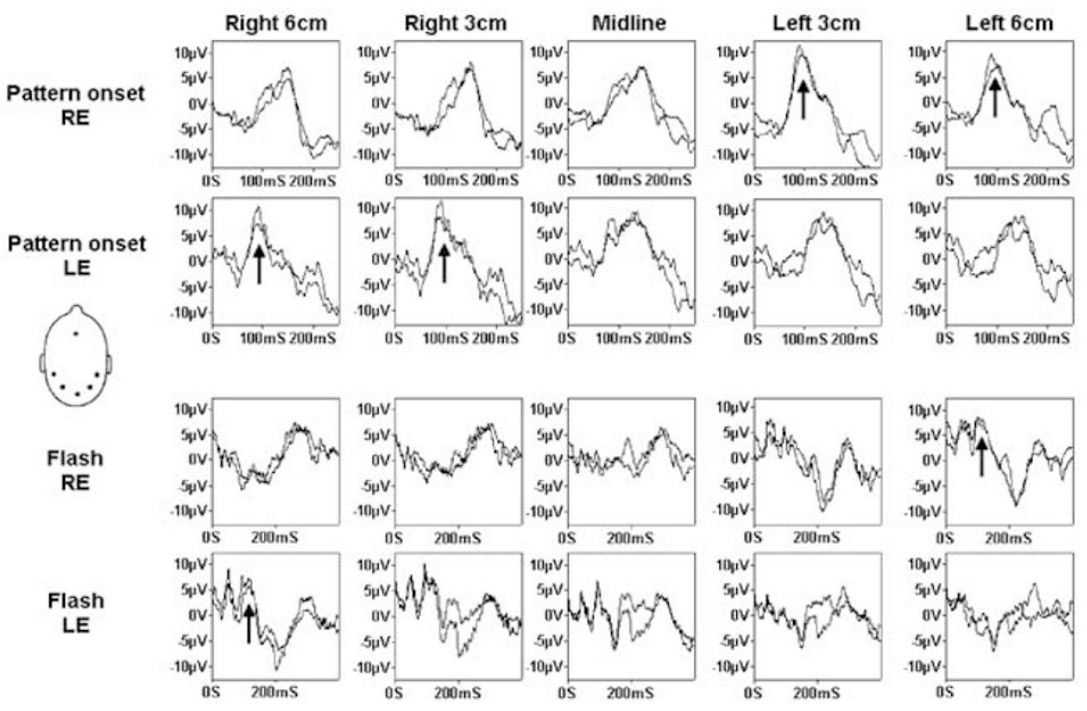

Figure 4 Pattern appearance (150 ms onset) and flash VEPs in a 7.5-year-old girl with the intracranial misrouting of albinism. Note the pronounced contralateral predominance of the VEPs to both pattern and flash stimuli (arrows). Pattern appearance VEPs only show the positive component at $100 \mathrm{~ms}$ in the contralateral hemisphere traces. The flash VEPs show little activity in the first $100 \mathrm{~ms}$ in the ipsilateral hemisphere traces, but a clear FVEP in the contralateral traces. Source: Dorey et al. ${ }^{67}$ 
end-stage disease, but the PERG P50 was always detectable even when the pattern VEP was extinguished (Figure 3). A shortening of latency is presumed to reflect loss of the ganglion cell derived N95 component, with additional loss of that part of P50 arising in relation to ganglion function. The earlier, more distally generated part of P50 remains even in severe ganglion cell loss, giving apparent shortening of latency.

Patients with LHON tend to present acutely, and have completely normal P50 components with very poor N95 in keeping with primary ganglion cell pathology. DOA rarely presents with an acute event, and the N95 loss in DOA is assumed to be progressive based on examination of patients in different stages of disease. ${ }^{66}$ Diagnostically, it is often a symmetrical and marked reduction in the N95 component that suggests primary ganglion cell dysfunction rather than dysfunction consequent upon an optic nerve insult.

\section{Albinism}

Albinism is a disorder of melanin synthesis resulting in hypopigmentation. Although perhaps not strictly an
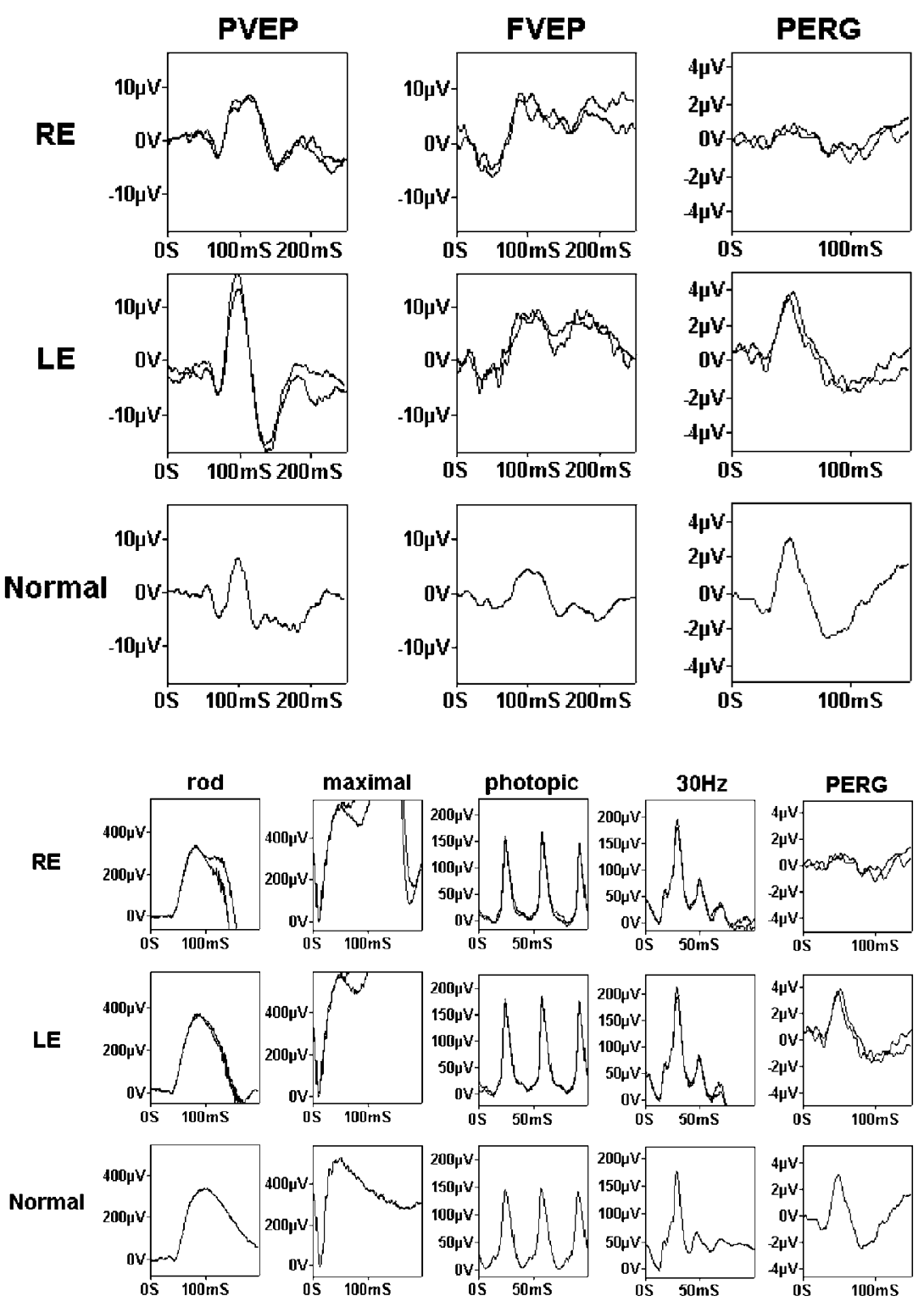

Figure 5 Electrophysiological findings in a 33-year-old female referred for investigation of presumed optic neuritis consequent upon dengue fever. There was a 2-month history of right eye visual acuity loss, but no signs other than a questionable right relative afferent pupillary defect. Note the delayed right eye pattern VEP, but the marked P50 component amplitude reduction suggests macular rather than optic nerve dysfunction. Full-field ERGs are normal, confirming that the dysfunction is confined to the macular rather than being part of generalised retinal dysfunction. The spatial extent of the loss of macular function is revealed by mfERG. 


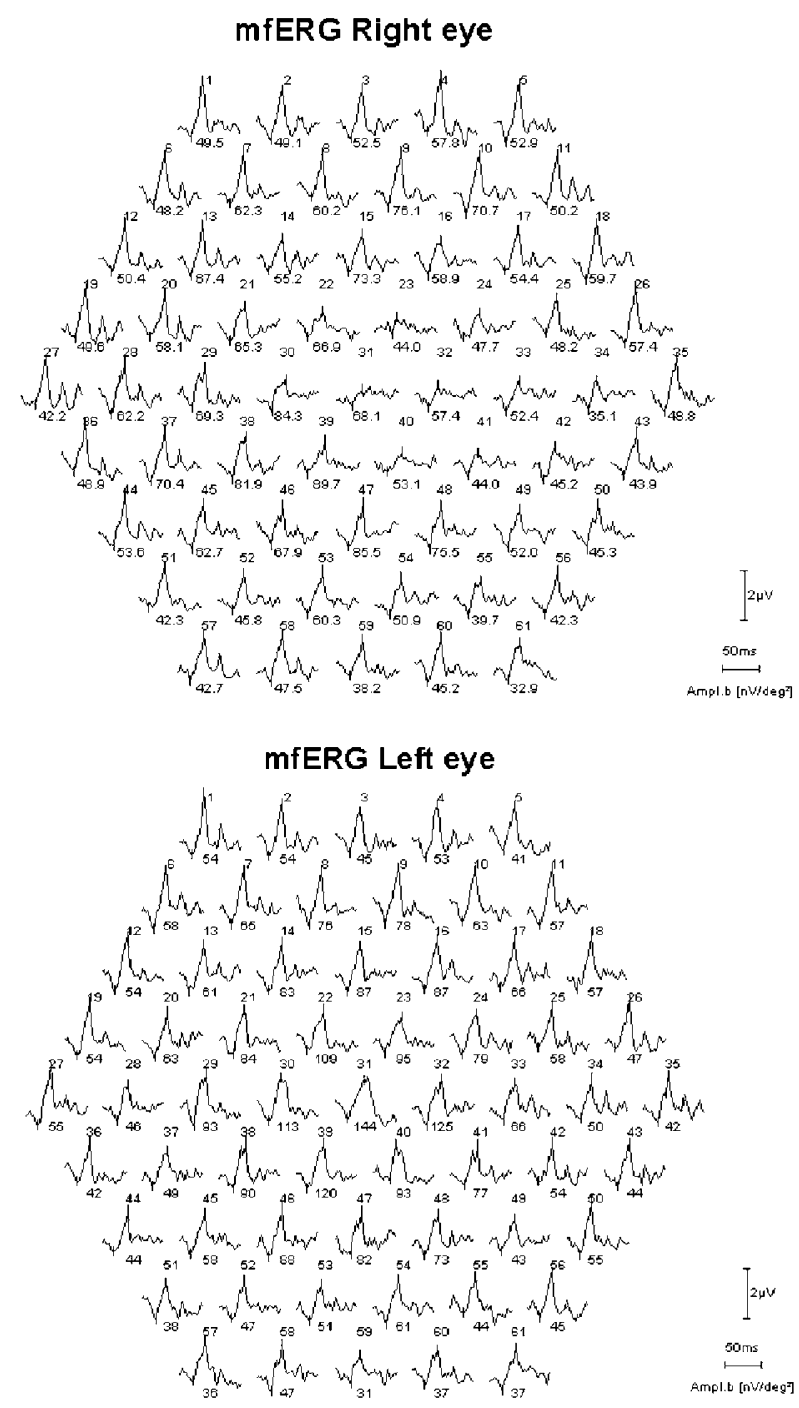

Figure 5 continued.

optic nerve disease, patients with albinism have an abnormal misrouting of optic nerve fibres such that the majority of optic nerve fibres from each eye project to the contralateral hemisphere. In a normally pigmented individual approximately $50 \%$ of optic nerve fibres project to the ipsilateral hemisphere, and approximately $50 \%$ to the contralateral hemisphere. There are two basic forms of albinism, oculocutaneous, where there is hypopigmentation of the skin and hair in addition to ocular signs, and ocular, where the signs are restricted to the visual system, and comprise nystagmus, foveal hypoplasia, fundal hypopigmentation, and iris transillumination. Not all patients display all signs, and recent work points to albinism being a spectrum of disease rather than an 'all or nothing' phenomenon. ${ }^{67}$ Although the diagnosis is not usually difficult in oculocutaneous albinism, the absence of cutaneous signs in ocular albinism does not facilitate diagnosis. However, the albinoid misrouting present in both forms may readily be demonstrated by VEP recording using multiple channel VEP recording from multiple posteriorly situated electrodes. ${ }^{67,68}$ (see Figure 4). In adults, the abnormality is best demonstrated using a pattern appearance stimulus; in younger patients a diffuse flash stimulus is effective. ${ }^{67,69}$ Indeed, it has recently been reported that there are dynamic changes occurring in the intracranial visual pathways of albinos such that flash VEPs normalise with age, but the pattern appearance VEPs become more abnormal with advancing years. ${ }^{70}$

\section{Other optic nerve disorders}

Toxic or nutritional optic nerve dysfunction manifests the expected electrophysiological abnormalities. There is VEP abnormality, usually involving both amplitude reduction and latency delay, and there may be associated involvement of the N95 component of the PERG.

Examples include ethambutol toxicity and tobaccoalcohol-related optic neuropathy. ${ }^{71}$

\section{On the distinction between optic nerve and macular dysfunction}

Previous authors have addressed some of the clinical difficulties that may be encountered in distinguishing between optic nerve and macular dysfunction. ${ }^{72,73}$ Visual acuity and colour vision loss are features of both, and a relative afferent pupillary defect, although often associated with optic nerve dysfunction, may also occur in macular disease. ${ }^{74-76}$ Equally, a delayed VEP is commonplace in macular dysfunction (Figures 5 and 6), and a delayed pattern VEP should not be assumed necessarily to reflect optic nerve disease in a symptomatic patient until it has adequately been demonstrated that macular function is normal. The finding of a delayed PVEP should therefore initiate further testing of macular function. This is probably best performed with PERG, but mfERG may also identify macular dysfunction (eg Figure 5). Note that even relatively mild PVEP delay is associated with marked reduction in PERG P50 in patients with macular dysfunction (Figure 6).

The PERG has been shown to play a crucial role in unexplained visual acuity loss, either where the fundus is normal or where there is disc pallor unaccompanied by vessel attenuation, visible abnormality at the macula or pigment migration; or when there are only mild signs but considerable visual loss. ${ }^{1}$ An undetectable PERG is not a feature even of severe optic nerve disease. ${ }^{2}$ Marked P50 amplitude reduction is uncommon in optic nerve dysfunction, and usually occurs only in severe disease 


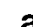

a

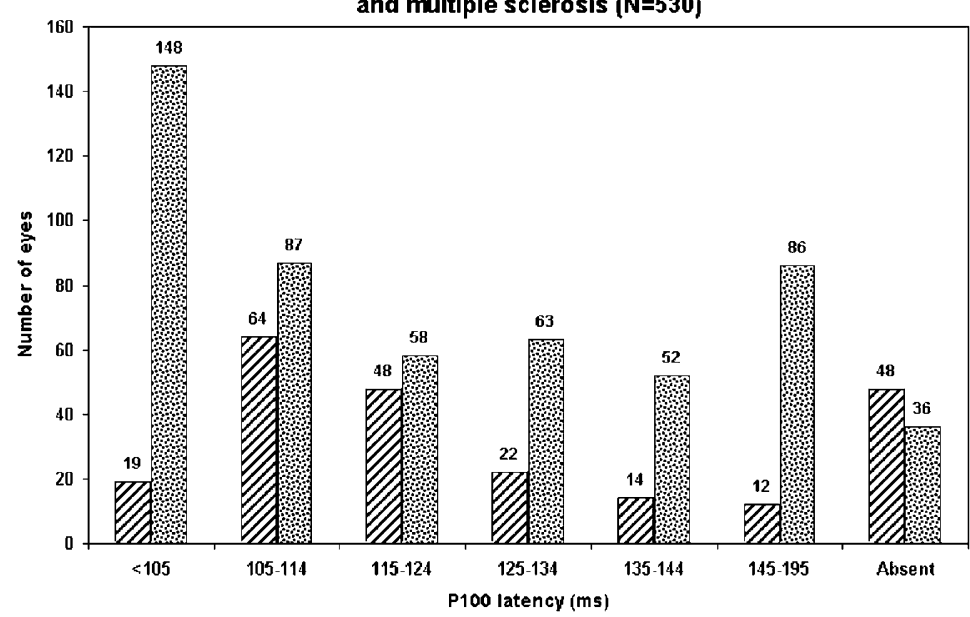

b PVEP latency vs PERG P50 in macular dysfunction ( $N=227$ )

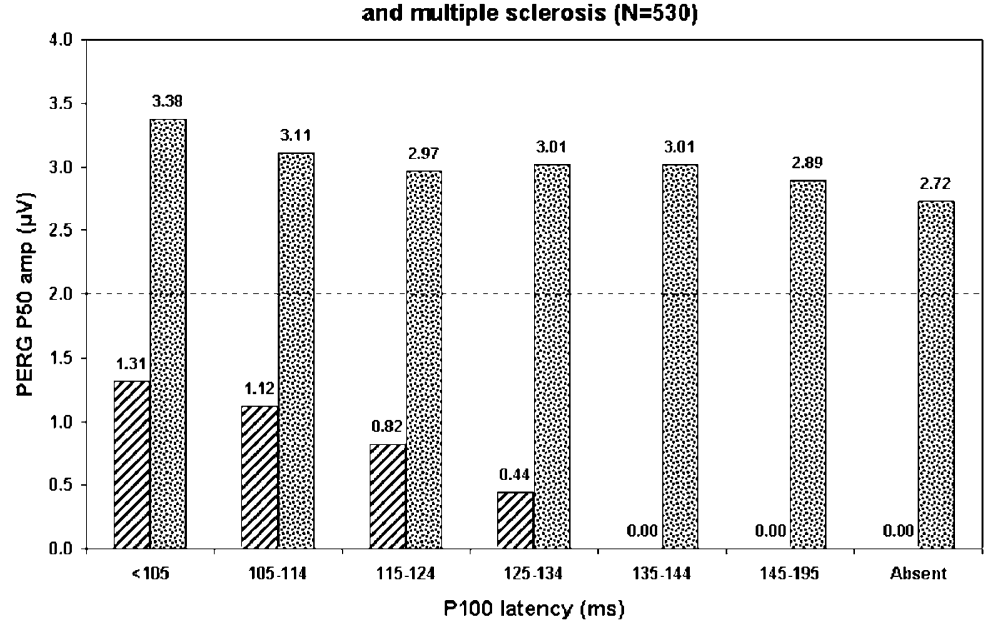

Figure 6 (a) Histogram demonstrating the incidence of PVEP delay in macular dysfunction and multiple sclerosis. All values $>105 \mathrm{~ms}$ are abnormal. Note the high incidence of PVEP P100 delay in macular dysfunction, with $>50 \%$ of eyes having a VEP delay $>10 \mathrm{~ms}$. The number of eyes in each histogram bin is indicated. Source: Holder. ${ }^{1}$ (b) Mean PERG P50 component amplitude for the patients shown in (a) against PVEP P100 component latency. P100 latency $>105 \mathrm{~ms}$ is abnormal for the laboratory. No eye with macular disease and P100 latency greater than $135 \mathrm{~ms}$ still has a detectable PERG. Mean P50 amplitude in patients with a latency of $125-134 \mathrm{~ms}$ is $<0.5 \mu \mathrm{V}$ in macular disease, but $\sim 3.0 \mu \mathrm{V}$ in optic nerve demyelination. The normal latency values shown in the multiple sclerosis group reflect the 'normal' eye in patients with monocular VEP delay. Note that even in eyes with no detectable PVEP the P50 component remains well within the normal range in the patients with optic nerve dysfunction. Source: Holder. ${ }^{1}$

accompanied by shortening of latency. An undetectable PERG, or severe reduction unaccompanied by P50 latency shortening, thus indicates macular dysfunction. In contrast, the PERG may be normal in optic nerve disease, or may show amplitude reduction confined to N95, an almost invariably present observation in primary ganglion cell dysfunction.

\section{Concluding remarks}

Electrophysiological investigation is a powerful tool in the objective evaluation of optic nerve and intracranial visual pathway function. Clinically, it is used alongside the structural information provided by neuroradiological investigation to give a more complete assessment in an individual patient. The VEP is a particularly useful tool, and can demonstrate optic nerve demyelination unaccompanied by signs or symptoms. However, VEP abnormalities are nonspecific, and an adjunctive test of macular function is required before a delayed or otherwise abnormal VEP in a patient with visual symptoms can be assumed to reflect optic nerve rather than macular dysfunction. Multifocal ERG can also be used, but the objective assessment of retinal ganglion cell dysfunction directly provided by the N95 component of the PERG, and the fact that the PERG is elicited by a stimulus directly 
comparable to that used for the pattern VEP, suggests that the PERG is the more appropriate parameter.

\section{References}

1 Holder GE. The pattern electroretinogram and an integrated approach to visual pathway diagnosis. Prog Retin Eye Res 2001; 20: 531-561.

2 Holder GE. The pattern electroretinogram. In: Fishman GA, Birch DG, Holder GE, Brigell MG (eds). Electrophysiologic Testing in Disorders of the Retina, Optic Nerve, and Visual Pathway, 2nd ed. Ophthalmology Monograph 2. The Foundation of the American Academy of Ophthalmology: San Francisco, CA, 2001.

3 Holder GE, Robson AG, Hogg CR, Kurz-Levin M, Lois N, Bird AC. Pattern ERG: clinical overview, and some observations on associated fundus autofluorescence imaging in inherited maculopathy. Doc Ophthalmol 2003; 106: 17-23.

4 Bach M, Hawlina M, Holder GE, Marmor MF, Meigen T, Vaegan et al. Standard for pattern electroretinography. Doc Ophthalmol 2000; 101: 11-18.

5 Holder GE. The significance of abnormal pattern electroretinography in anterior visual pathway dysfunction. Br J Ophthalmol 1987; 71: 166-171.

6 Viswanathan S, Frishman LJ, Robson JG. The uniform field and pattern ERG in macaques with experimental glaucoma: removal of spiking activity. Invest Ophthalmol Vis Sci 2000; 41: 2797-2810.

7 Bush RA, Sieving PA. A proximal retinal component in the primate photopic ERG a-wave. Invest Ophthalmol Vis Sci 1994; 35: 635-645.

8 Shiells RA, Falk G. Contribution of rod, on-bipolar, and horizontal cell light responses to the ERG of dogfish retina. Vis Neurosci 1999; 16: 503-511.

9 Green DG, Kapousta-Bruneau NV. A dissection of the electroretinogram from the isolated rat retina with microelectrodes and drugs. Vis Neurosci 1999; 16: 727-741.

10 Lei B, Perlman I. The contributions of voltage- and time-dependent potassium conductances to the electroretinogram in rabbits. Vis Neurosci 1999; 16: 743-754.

11 Marmor MF, Holder GE, Seeliger M, Yamamoto S. Standard for clinical electroretinography. Doc Ophthalmol 2004, in press.

12 Sutter EE, Tran D. The field topography of ERG components in man - I. The photopic luminance response. Vision Res 1992; 32: 433-446.

13 Hood DC. Assessing retinal function with the multifocal technique. Prog Retin Eye Res 2000; 19: 607-646.

14 Richey ET, Kooi KA, Tourtellotte WW. Visually evoked responses in multiple sclerosis. J Neurol Neurosurg Psychiatry 1971; 34: 275-280.

15 Halliday AM, McDonald WI, Mushin J. Delayed visual evoked response in optic neuritis. Lancet 1972; i: 982-985.

16 Halliday AM, McDonald WI, Mushin J. Visual evoked response in diagnosis of multiple sclerosis. BMJ 1973; 4: 661-664.

17 Holder GE. Multiple sclerosis. In: Heckenlively JR, Arden GB (eds). Principles and Practice of Clinical Electrophysiology of Vision. Mosby Year Book: St Louis, MO, 1991 pp 797-805.

18 Jones SJ, Brusa A. Neurophysiological evidence for longterm repair of MS lesions: implications for axon protection. J Neurol Sci 2003; 206: 193-198.

19 Holder GE. The incidence of abnormal pattern electroretinography in optic nerve demyelination. Electroencephalogr clin Neurophysiol 1991; 78: 18-26.
20 Berninger TA, Heider W. Pattern electroretinograms in optic neuritis during the acute stage and after remission. Graefe's Arch Clin Exp Ophthalmol 1990; 228: 410-414.

21 Youl BD, Turano G, Miller DH, Towell AD, MacManus DG, Moore SG et al. The pathophysiology of acute optic neuritis. An association of gadolinium leakage with clinical and electrophysiological deficits. Brain 1991; 114: 2437-2450.

22 Hickman SJ, Brierley CM, Brex PA, MacManus DG, Scolding NJ, Compston DA et al. Continuing optic nerve atrophy following optic neuritis: a serial MRI study. Mult Scler 2002; 8: 339-342.

23 Hood DC, Odel JG, Zhang X. Tracking the recovery of local optic nerve function after optic neuritis: a multifocal VEP study. Invest Ophthalmol Vis Sci 2000; 41: 4032-4038.

24 Rinalduzzi S, Brusa A, Jones SJ. Variation of visual evoked potential delay to stimulation of central, nasal, and temporal regions of the macula in optic neuritis. J Neurol Neurosurg Psychiatry 2001; 70: 28-35.

25 Wilson WB. Visual evoked response differentiation of ischaemic optic neuritis from the optic neuritis of multiple sclerosis. Am J Ophthalmol 1978; 86: 530-535.

26 Hennerici M, Wenzel D, Freund HJ. The comparison of small size rectangle and checkerboard stimulation for the evaluation of delayed visual evoked responses in patients suspected of multiple sclerosis. Brain 1977; 100: 119-136.

27 Glaser JS, Laflamme P. The visual evoked response: methodology and application in optic nerve disease. In: Thompson HS (ed). Topics in Neuro-ophthalmology. Williams \& Wilkins: Baltimore, MD, 1979, pp 199-218.

28 Harding GFA, Crews SJ, Good PA. VEP in neuroophthalmic disease. In: Barber C (ed). Evoked Potentials.. MTP Press Ltd: Lancaster, England, 1980, pp 235-241.

29 Holder GE. The visual evoked potential in ischaemic optic neuropathy. Doc Ophthalmol Proc Ser 1981; 27: 123-129.

30 Cox TA, Thompson HS, Hayreh SS, Snyder JE. Visual evoked potential and pupillary signs. Arch Ophthalmol 1982; 100: $1603-1607$

31 Wildberger H. Pattern-evoked potentials and visual field defects in ischaemic optic neuropathy. Doc Ophthalmol Proc Ser 1984; 40: 193-201.

32 Lehmann D, Skrandies W. Visually evoked scalp potential fields in hemiretinal stimulation. Doc Ophthalmol Proc Ser 1980; 23: 237-243.

33 Thompson PD, Mastaglia FL, Carroll WM. Anterior ischaemic optic neuropathy. A correlative clinical and visual evoked potential study of 18 patients. J Neurol Neurosurg Psychiatry 1986; 49: 128-135.

34 Holder GE. Ischaemic optic neuropathy. In: Heckenlively JR and Arden GB (eds). Principles and Practice of Clinical Electrophysiology of Vision. Mosby Year Book: St Louis, MO, 1991, pp 636-639.

35 Almarcegui C, Dolz I, Alejos MV, Fernandez FJ, Valdizan JR, Honrubia FM. Pattern electroretinogram in anterior ischemic optic neuropathy. Rev Neurol 2001; 32: 18-21.

36 Salazar JJ, Ramirez AI, De Hoz R, Triviño A, Ramirez JM. Apoptosis in optical ischaemic neuropathy. Abstracts, EVER Meeting, Palma 1999.

37 Muller W. Untersuchungen uber das Verhalten der Corticalzeit bei bitemporaler Hemianopsie. Graefes Arch Ophthalmol 1962; 165: 214-218.

38 Vaughan HG, Katzman R, Taylor J. Alterations of visual evoked response in the presence of homonymous visual defects. Electroencephalogr Clin Neurophysiol 1963; 15: 737-746. 
39 Jacobsen JH, Hirose T, Suziki TA. Simultaneous ERG and VER in lesions of the optic pathway. Invest Ophthalmol 1968; 7: 279-292.

40 Kooi KA, Yamada T, Marshall RE. Field studies of monocularly evoked cerebral potentials in bitemporal hemianopsia. Neurology 1973; 23: 1217-1225.

41 Wildberger HG, Van Lith GH, Wijngaarde R, Mak GT. Visually evoked cortical potentials in the evaluation of homonymous and bitemporal visual field defects. $\mathrm{Br} \mathrm{J}$ Ophthalmol 1976; 60: 273-278.

42 Halliday AM, HallidayE, Kriss A et al. The pattern evoked potential in compression of the anterior visual pathways. Brain 1976; 99: 357-374.

43 Blumhardt LD, Barrett G, Halliday AM. The asymmetrical visual evoked potential to pattern reversal in one half field and its significance for the analysis of visual field defects. Br J Ophthalmol 1977; 61: 454-461.

44 Holder GE. The effects of chiasmal compression on the pattern visual evoked potential. Electroencephalogr Clin Neurophysiol 1978; 45: 278-280.

45 Holder GE, Bullock PR. Visual evoked potentials in the assessment of patients with non-functioning chromophobe adenomas. J Neurol Neurosurg Psychiatry 1989; 52: 31-37.

46 Gott PS, Weiss MH, Apuzzo M, van der Meulen JP. Checkerboard visual evoked response in evaluation and management of pituitary tumours. Neurosurgery 1979; 5: 553-558.

47 Stark DJ, Lenton L. Electrophysiological assessment of compressive lesions of anterior visual pathways. Aust J Ophthalmol 1981; 9: 135-141.

48 Haimovic IC, Pedley TA. Hemi-field pattern reversal visual evoked potentials. II. Lesions of the chiasm and posterior visual pathways. Electroencephalogr Clin Neurophysiol 1982; 54: 121-131.

49 Maitland CG, Aminoff MJ, Kennard C, Hoyt WF. Evoked potentials in the evaluation of visual field defects due to chiasmal or retrochiasmal lesions. Neurology 1982; 32: 986-991.

50 Onofrj M, Bodis-Wollner I, Mylin L. Visual evoked potential diagnosis of field defects in patients with chiasmatic and retrochiasmatic lesions. J Neurol Neurosurg Psychiatry 1982; 45: 294-302.

51 Flanagan JG, Harding GFA. Multi-channel visual evoked potentials in early compressive lesions of the optic chiasm. Doc Ophthalmol 1987; 69: 271-282.

52 Brecelj J. Electrodiagnostics of chiasmal compressive lesions. Int J Psychophysiol 1994; 16: 263-272.

53 Kaufman DI, Lorance RW, Woods M, Wray SH. The pattern electroretinogram: a long-term study in acute optic neuropathy. Neurology 1988; 38: 1767-1774.

54 Ruther K, Ehlich P, Philipp A, Eckstein A, Zrenner E. Prognostic value of the pattern electroretinogram in cases of tumors affecting the optic pathway. Graefes Arch Clin Exp Ophthalmol 1998; 236: 259-263.

55 Parmar DN, Sofat A, Bowman R, Bartlett JR, Holder GE. Prognostic value of the pattern electroretinogram in chiasmal compression. Br J Ophthalmol 2000; 84: 1024-1026.

56 Kjer P. Infantile optic atrophy with dominant mode of inheritance: a clinical and genetic study of 19 Danish families. Acta Ophthalmol 1959; 37(Suppl 54): 1-146.

57 Borruat FX, Green WT, Graham EM, Sweeney MG, Morgan-Hughes JA, Sanders MD. Late onset Leber's optic neuropathy: a case confused with ischaemic optic neuropathy. Br J Ophthalmol 1992; 76: 571-573.
58 Riordan-Eva P, Sanders MD, Govan GG, Sweeney MG, Da Costa J, Harding AE. The clinical features of Leber's hereditary optic neuropathy defined by the presence of a pathogenic mitochondrial DNA mutation. Brain 1995; 118: 319-337.

59 Johns DR, Newman NJ. Hereditary optic neuropathies. Semin Ophthalmol 1995; 10: 203-213.

60 Holder GE. The pattern electroretinogram in anterior visual pathway dysfunction and its relationship to the pattern visual evoked potential: a personal clinical review of 743 eyes. Eye 1997; 11: 924-934.

61 Hung HL, Kao LY, Huang CC. Clinical features of Leber's hereditary optic neuropathy with the 11778 mitochondrial DNA mutation in Taiwanese patients. Chang Gung Med J 2003; 26: 41-47.

62 Votruba M, Fitzke FW, Holder GE, Carter A, Bhattacharya SS, Moore AT. Clinical features in affected individuals from 21 pedigrees with dominant optic atrophy. Arch Ophthalmol 1998; 116: 351-358.

63 Johnston PB, Gaster RN, Smith VC, Tripathi RC. A clinicopathological study of autosomal dominant optic atrophy. Am J Ophthalmol 1979; 88: 868-875.

64 Kjer P. Histopathology of eye, optic nerve and brain in a case of dominant optic atrophy. Acta Ophthalmol 1982; 61: 300-312.

65 Berninger TA, Jaeger W, Krastel H. Electrophysiology and colour perimetry in dominant infantile optic atrophy. $\mathrm{Br} \mathrm{J}$ Ophthalmol 1991; 75: 49-52.

66 Holder GE, Votruba M, Carter AC, Bhattacharya SS, Fitzke FW, Moore AT. Electrophysiological findings in dominant optic atrophy (DOA) linking to the OPA1 locus on chromosome 3q28-qter. Doc Ophthalmol 1999; 95: 217-228.

67 Dorey SE, Neveu MM, Burton LC, Sloper JJ, Holder GE. The clinical features of albinism and their correlation with visual evoked potentials. Br J Ophthalmol 2003; 87: 767-772.

68 Apkarian P. A practical approach to albino diagnosis. VEP misrouting across the age span. Ophthalmic Paediatr Genet 1992; 13: 77-88.

69 Kriss A, Russell-Eggitt I, Harris CM, Lloyd IC, Taylor D. Aspects of albinism. Ophthalmic Paediatr Genet 1992; 13: 89-100.

70 Neveu MM, Jeffery G, Burton LC, Sloper JJ, Holder GE. Age-related changes in the dynamics of human albino visual pathways. Eur J Neurosci 2003; 18: 1939-1949.

71 Srivastava AK, Goel UC, Bajaj S, Singh KJ, Dwivedi NC, Tandon MP. Visual evoked responses in ethambutol induced optic neuritis. J Assoc Physicians India 1997; 45: 847-849.

72 Sadun AA. Distinguishing between clinical impairments due to optic nerve or macular disease. Metab Pediatr Syst Ophthalmol 1990; 13: 79-84.

73 Newman NJ. Optic disc pallor: a false localizing sign. Surv Ophthalmol 1993; 37: 273-282.

74 Thompson HS, Warsky RC, Weinstein JM. Pupillary dysfunction and macular disease. Trans Am Ophthalmol Soc 1980; 78: 311-317.

75 Newsome DA, Milton RC, Gass JD. Afferent pupillary defect in macular degeneration. Am J Ophthalmol 1981; 92: 396-402.

76 Folk JC, Thompson HS, Han DP, Brown CK. Visual function abnormalities in central serous retinopathy. Arch Ophthalmol 1984; 102: 1299-1302. 